\title{
Polymer Composites with Oriented Magnetic Nanowires as Fillers
}

\author{
Li Sun, and Kusuma Keshoju \\ Department of Mechanical Engineering, University of Houston, Houston, TX, 77204-4006
}

\begin{abstract}
Metallic nickel nanowires with excellent physical properties have been introduced into polydimethylsiloxane (PDMS) matrix to form polymer nanocomposites. Nanowires were synthesized by template-assisted electrochemical deposition. By utilizing ferromagnetic nickel nanowires, small external magnetic field can be used to control their alignment and distribution during composite synthesis. Unlike dielectrophoresis, optical tweezers, and microfluidic flow control, magnetic manipulation provides a cost-effective, non-contact, and versatile approach to control nanostructured materials in fluids over a large area. Polydimethylsiloxane composites with nanowires arranged in longitudinal, transverse, and random orientations with respect to the applied load direction were studied. Tensile tests showed that the composites with longitudinal arrangement have higher elastic modulus and tensile strength than the other composite samples. Experimentally obtained elastic modulus values were compared with the prediction of classical Halpin-Tsai model.
\end{abstract}

\section{INTRODUCTION}

Polymer nanocomposites is a rapidly growing area in nano-engineered materials research. The use of nanoscale fillers to augment the properties of polymers has led to the development of nanocomposites and has provided a radical alternative to the conventional polymer composite systems. Nanocomposites can possess a combination of properties that cannot be achieved with either of the constituents acting alone [1]. In this study, we have explored the synthesis and mechanical characterization of polymer-nickel (Ni) nanowire composites. The nanowires were synthesized by template-assisted electrodeposition [2], in which a desired material is electrochemically deposited inside the nanopores of template and shaped into a nanowire with controlled dimensions and composition.

Manipulation of nanomaterials is not only an engineering challenge, but can also help in understanding the nanoscale physics problems. To improve the effectiveness of manipulation, most of the experiments were performed on nanomaterials suspended in fluids. Techniques based on Langmuir-Blodgett film formation [3], microfluidic flow [4], electric [5] and magnetic [6] fields, dielectrophoresis [7], and optical tweezers [8] have been explored. Among all these methods, magnetic manipulation provides a cost-effective, non-contact, and versatile approach to control nanostructured materials in fluids over a large area. The ferromagnetic materials can be effectively manipulated with very small external magnetic field and the magnetic interaction can be three or four orders of magnitude stronger than any other interactions [9]. 
Based on the analysis of magnetic driving field and fluid drag force, the motion of nanowires can be well quantified [10]. The schematic of the geometry during nanowire rotation is shown in Figure 1(a). The dotted line represents the original nanowire position at time, $t=0$. The nanowire and field angles are defined by $\theta_{w}(t)$ and $\theta_{H}(t)$. At any instant of time, lag angle $\theta_{l}(t)$ is defined as the angle between wire and field.
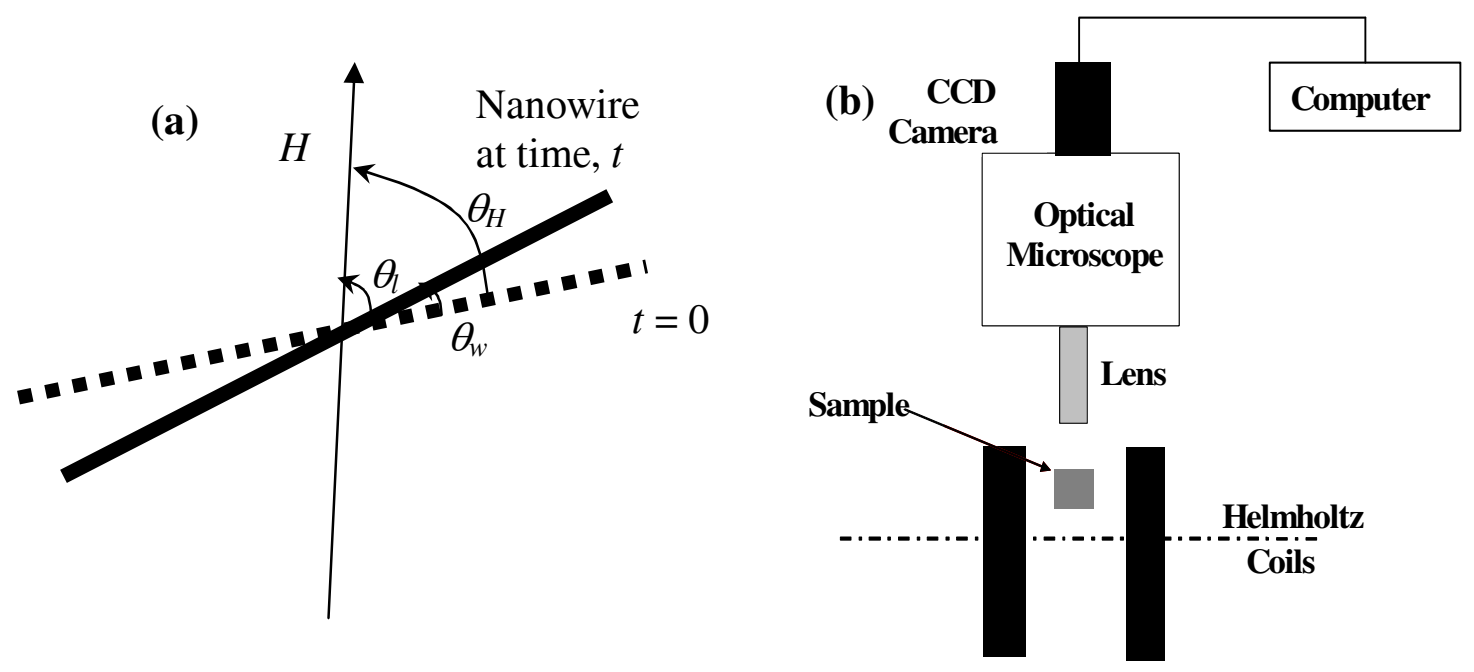

Figure 1. (a) Angular relationships between nanowire and external field $(H)$ during alignment. (b) Schematic drawing of the magnetic manipulation setup.

We have chosen polydimethylsiloxane (PDMS) as the polymer matrix. PDMS is nontoxic, biocompatible, and easy to process. It is an elastomer with high ductility but has low elastic modulus and tensile strength. Nanofillers such as silica and carbon black have been used to reinforce PDMS [11,12]. Here we demonstrate the synthesis of PDMS-Ni composites with controlled nanowire alignment. Anisotropic mechanical properties of these composites were studied.

The level of reinforcement of polymers depends on the concentration, distribution and orientation of nanowires. The composite will exhibit isotropic properties with random distribution of nanowires. In contrast, aligned composite samples show anisotropic properties, and mechanical responses depend on the angle between the applied load and the nanowire alignment direction. Halpin-Tsai model [13] describes the elastic properties of composite in terms of the fiber (nanowire) orientations as well as the elastic properties of the fiber and the polymer matrix. The longitudinal and transverse configurations are defined when the fibers are arranged in parallel and perpendicular to the applied load direction, respectively. Elastic modulus of composite with fibers arranged in longitudinal $\left(E_{L}\right)$, transverse $\left(E_{T}\right)$ and random $\left(E_{R}\right)$ configurations are given by [13]

$$
\begin{aligned}
& E_{L}=\frac{1+2\left(l_{f} / d_{f}\right) \eta_{L} v_{f}}{1-\eta_{L} v_{f}} E_{m}, \\
& E_{T}=\frac{1+2 \eta_{T} v_{f}}{1-\eta_{L} v_{f}} E_{m},
\end{aligned}
$$




$$
\begin{aligned}
\eta_{L} & =\frac{\left(E_{f} / E_{m}\right)-1}{\left(E_{f} / E_{m}\right)+2\left(l_{f} / d_{f}\right)} \text { and } \eta_{T}=\frac{\left(E_{f} / E_{m}\right)-1}{\left(E_{f} / E_{m}\right)+2}, \\
E_{R} & =\frac{3}{8} E_{L}+\frac{5}{8} E_{T},
\end{aligned}
$$

where $l_{f}, d_{f}, v_{f}$, and $E_{f}$ are the length, diameter, volume fraction, and elastic modulus of fibers, respectively and $E_{m}$ is the elastic modulus of the matrix.

\section{EXPERIMENT}

$\mathrm{Ni}$ nanowires were fabricated by template-assisted electrodeposition. A nanoporous alumina template was used as a working electrode inside which the $\mathrm{Ni}$ nanowires were deposited. More experimental details can be found elsewhere [14]. The PDMS material supplied by Dow Corning comprised of a curing agent and a base solvent. The two parts were mixed at a 10:1 volume ratio using a mechanical stirrer. Vacuum degassing was used to remove air in the mixture. For composite fabrication, dry Ni nanowires were mixed with uncured PDMS. PDMS$\mathrm{Ni}$ composite was then spin coated to make thin films. Both pure PDMS and composite films were allowed to cure at room temperature. After curing, the films were cut into dog-bone shaped tensile specimens. The middle portion of the sample has a cross-sectional area of $1.27 \times 0.3175$ $\mathrm{cm}^{2}$. The thickness of the samples ranges from 100-300 $\mu \mathrm{m}$. Composite films with different nanowire concentrations and orientations were prepared. The films were tested in the customdesigned tensile testing machine. Stress-strain curves were plotted. Elastic modulus and tensile strength were quantified as a function of orientation and concentration of nanowires.

Alignment of Ni nanowires was obtained by placing the spin coated films in the uniform magnetic field $\left(H=4.62 \times 10^{-4} \mathrm{~T}\right)$ generated by a set of Helmholtz coils. Figure $1(\mathrm{~b})$ shows the schematic drawing of the experimental setup for observation of nanowire alignment along the magnetic field. Magnetic field has a uniformity of $0.5 \%$ in a cylindrical volume of one inch long and one inch in diameter. The nanowire motion was observed by camera which was connected to an optical microscope and a computer. Variation of the lag angle between wire and field with time was plotted.

\section{DISCUSSION}

Figure 2(a) is the scanning electron microscopy (SEM) image of Ni nanowires fabricated in the alumina template after being extracted from the template matrix. The nanowires have an average length of $40 \mu \mathrm{m}$ and diameter in the range of 100-200 $\mathrm{nm}$. Due to their length, the nanowires and their motion can be easily resolved under the optical microscope (the diameter of nanowires is below the optical resolution). Figure 2(b) shows the effect of magnitude of magnetic field on the alignment of nanowires in PDMS. As the magnitude of field increases, driving magnetic torque increases and alignment time decreases. Nanowires respond to the torques produced by the field and align in the direction of field. The lag angle between wire and field becomes zero after alignment. The transparency of PDMS allowed us to control the focus depth inside the bulk composite samples to monitor nanowire alignment and distribution across the entire sample. 

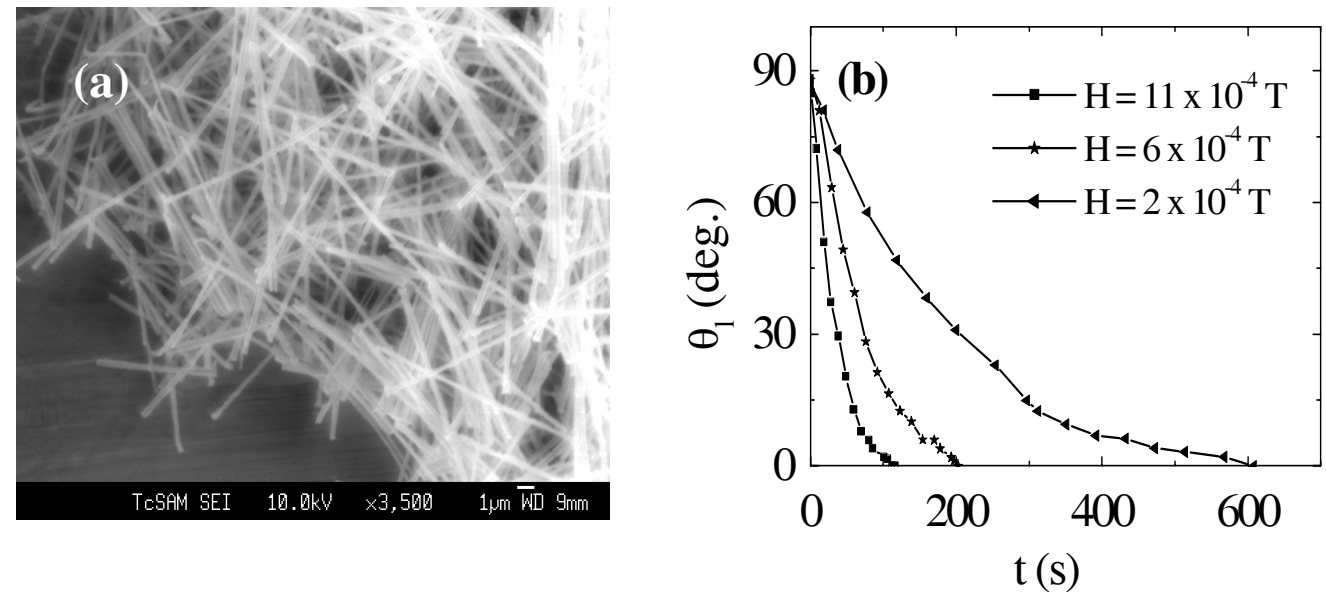

Figure 2. (a) SEM image of Ni nanowires. (b) Alignment of Ni nanowire in PDMS under different applied fields.

The optical micrograph of cured composite sample after magnetic alignment shows that the nanowires are well aligned and dispersed (Figure 3(a)). The stress-strain curves of composites with nanowires $(4.31 \mathrm{wt} . \%)$ oriented in different directions are summarized in Figure 3(b). For comparison, both pure PDMS and composite's results are plotted together. It is evident that the mechanical properties strongly depend on the direction of measurement. The elastic moduli of pure PDMS, longitudinal, transverse, and random composites are 1.2, 3, 1.1, and 1.67 $\mathrm{MPa}$, respectively. The corresponding tensile strength values are 1.79, 2.4, 1.64, and 1.8 $\mathrm{MPa}$, respectively. The longitudinal modulus is always greater than the random and transverse moduli. Nanowires contribute more to the development of the longitudinal modulus and the polymer matrix contributes more to the development of the transverse modulus. When a transverse load is applied to the composite, nanowires act as inclusions in the matrix instead of load carrying members. Random modulus is intermediate between longitudinal and transverse moduli. With random orientation of nanowires, it is possible to obtain equal mechanical properties in all directions in the plane of the composite.

Table I shows the comparison of elastic modulus of PDMS composites with the prediction of Halpin-Tsai model. In some cases, there is a disagreement between experimental and calculated values. This is because Halpin-Tsai equations are derived assuming perfect bonding and distribution of fibers.

Table I. Comparison of experimental elastic modulus of composites with the prediction of theoretical values calculated using Halpin-Tsai equations.

\begin{tabular}{|l|l|l|l|l|}
\hline \multirow{2}{*}{$\begin{array}{l}\text { Ni } \\
\text { nanowires } \\
\text { wt.\% }\end{array}$} & \multicolumn{2}{|l|}{$\begin{array}{l}\text { Longitudinal modulus, } \boldsymbol{E}_{\boldsymbol{L}} \\
(\mathrm{MPa})\end{array}$} & $\begin{array}{l}\text { Random modulus, } \boldsymbol{E}_{\boldsymbol{R}} \\
(\mathrm{MPa})\end{array}$ \\
\cline { 2 - 5 } & Experimental & Theoretical & Experimental & Theoretical \\
\hline 0.18 & 2.62 & 1.91 & 1.837 & 1.747 \\
\hline 1.0 & 2.69 & 3.03 & 2.08 & 2.16 \\
\hline 4.31 & 3 & 5.58 & 1.667 & 2.3 \\
\hline
\end{tabular}



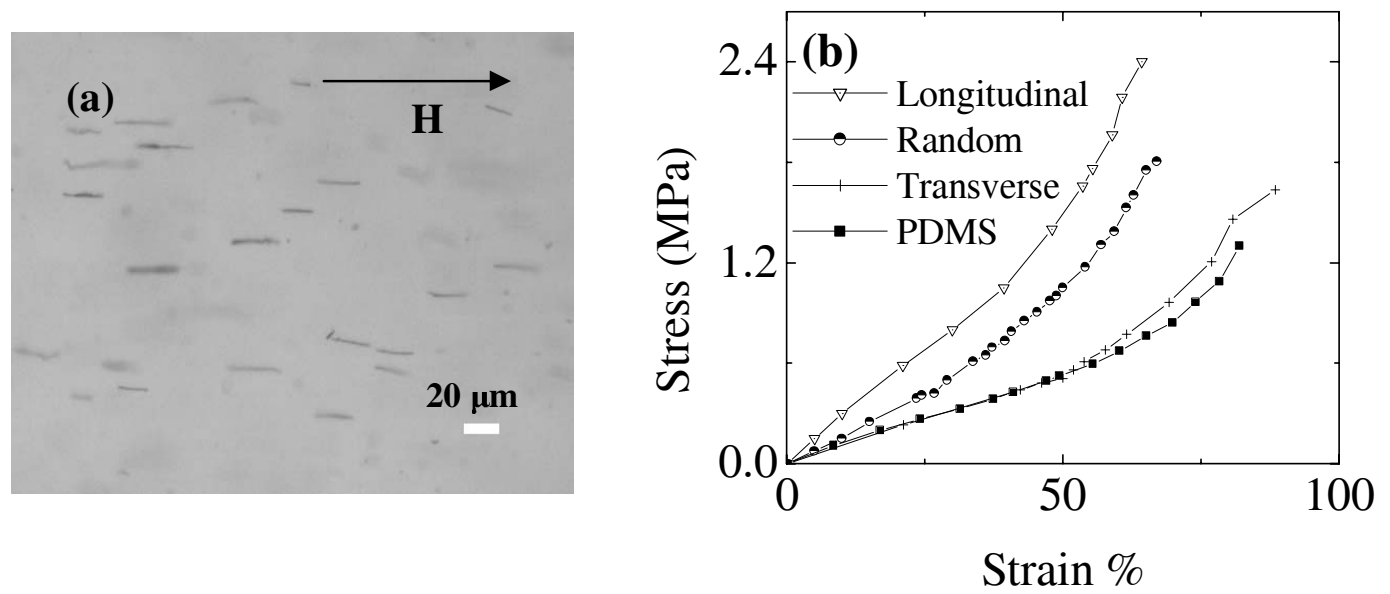

Figure 3. (a) Optical micrograph of Ni nanowires (0.18 wt.\%) aligned in PDMS along the magnetic field. (b) Stress-strain curves of PDMS composites with Ni nanowires arranged in different orientations.

As the weight fraction of nanowires increased, the elastic modulus of longitudinal composites increased due to the stiffening effect of nanowires (Figure 4(a)). This is possible when there is good dispersion and uniform distribution of nanowires, which in turn increases the adhesion between PDMS and Ni nanowires. Figure 4(b) shows the SEM image of fractured composite surface. The pulled out nanowires were covered with a $100 \mathrm{~nm}$ polymer sheath. This reveals failure of polymer rather than the polymer-nanowire interface indicating an excellent interfacial interaction between PDMS and nanowires. Therefore, nanowires are promising candidates for reinforcement of PDMS.
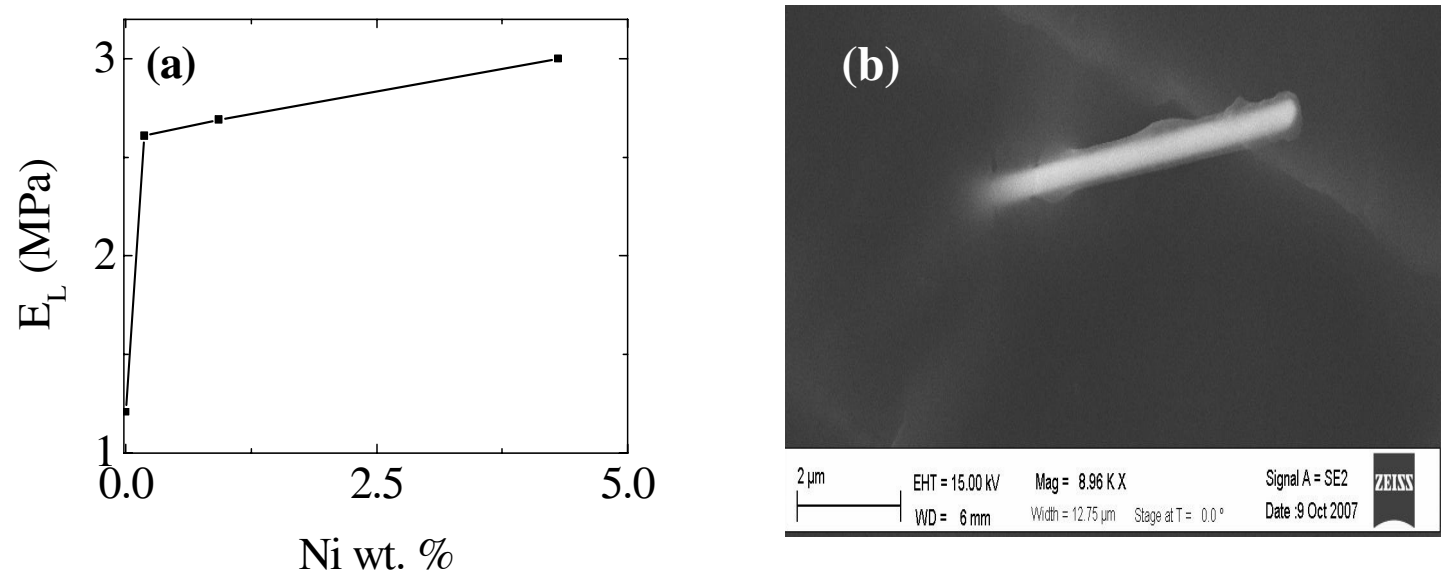

Figure 4. (a) Elastic modulus of longitudinal samples as a function of weight fraction of $\mathrm{Ni}$ nanowires in PDMS composites. (b) SEM image of fractured composite surface. 


\section{CONCLUSIONS}

PDMS composites reinforced with Ni nanowires have been fabricated. Nanowires were aligned in different orientations using external magnetic field. Magnetic field is a low-cost and highly efficient approach to manipulate nanowires in fluids. Composite samples with longitudinal arrangement of nanowires showed higher elastic modulus and tensile strength compared to random and transverse samples. Elastic modulus of longitudinal samples increased with weight fraction of $\mathrm{Ni}$ nanowires. These composite samples can have applications in protective coatings and sensor development.

\section{ACKNOWLEDGMENTS}

The authors thank financial support from TcSUH, AFOSR SPRING program and the Bill D. Cook Endowment.

\section{REFERENCES}

1. P. K. Mallick, Fiber-reinforced composites: materials, manufacturing and design, 2nd ed. (Marcel Dekker, New York, 1993), p. 2.

2. C. R. Martin, Science 266, 1961 (1994).

3. D. Whang, S. Jin, Y. Wu, and C. M. Lieber, Nano Lett. 3, 1255 (2003).

4. Y. Huang, X. F. Duan, Q. Q. Wei, and C. M. Lieber, Science 291, 630 (2001).

5. D. L. Fan, F. Q. Zhu, R. C. Cammarata, and C. L. Chien, Phys. Rev. Lett. 94, 247208 (2005).

6. M. Tanase, L. A. Bauer, A. Hultgren, D. M. Silevitch, L. Sun, D. H. Reich, P. C. Searson, and G. J. Meyer, Nano Lett. 1, 155 (2001).

7. S. Y. Lee, T. H. Kim, D. I. Suh, E. K. Suh, N. K. Cho, W. K. Seong, and S. K. Lee, Appl. Phys. A 87, 739 (2007).

8. P. Galajda and P. Ormos, Appl. Phys. Lett. 78, 249 (2001).

9. K. Keshoju and L. Sun, (unpublished).

10. K. Keshoju, L. Sun, and H. Xing, Appl. Phys. Lett. 91, 123114 (2007).

11. S. D. Burnside and E. P. Giannelis, J. Poly. Sci. B 38, 1595 (2000).

12. G. Kraus, Reinforcement of Elastomers, (Interscience Publishers, New York, 1965), p. 101.

13. J. C. Halpin and J. L. Kardos, Poly. Engg Sci. 16, 344 (1976).

14. K. Keshoju, X. F. Gu, A. T. Kumar, and L. Sun, Solid State Phen. 121-123, 839 (2007). 